\title{
LEVANTAMENTO DA SITUAÇÃO DO ACERVO DE RECURSOS DE INFORMAÇÃO DE UM LABORATÓRIO DE BIOQUÍMICA
}

\section{SURVEY OF THE SITUATION OF THE INFORMATION RESOURCES ACQUISITION OF A BIOCHEMISTRY LABORATORY}

\author{
Célia da Consolação Dias \\ Universidade Federal de Minas Gerais \\ Alessandra Conceição Faria Aguiar Campos \\ Universidade Federal de Minas Gerais \\ Sérgio Vale Aguiar Campos \\ Universidade Federal de Minas Gerais
}

\section{RESUMO}

Trata-se de um artigo cujo objetivo de apresentar detalhadamente a situação do LAB1 em relação à organização dos recursos de informações e de infraestrutura que estão disponíveis para o desenvolvimento das atividades de pesquisa de um laboratório de bioquímica. Este estudo foi realizado especificamente com uma visão direcionada para a gestão de informações deste laboratório buscando elementos e avaliando-os em relação aos documentos produzidos e recebidos, a situação em relação à gestão dos dados, a gestão dos recursos informacionais, dificuldades e facilidades da equipe interna para lidar com o conhecimento produzido, com os insumos e amostras utilizadas nos experimentos realizados diariamente pelos pesquisadores e, principalmente na verificação de como se dá o registro das informações e à sua recuperação para gerar novos conhecimentos. Para este estudo, foram utilizados os seguintes procedimentos metodológicos: levantamento bibliográfico, a definição do instrumento de coleta de dados; a coleta de dados e a organização dos dados coletados. Concluiu-se que pelo diagnóstico realizado pode-se perceber a necessidade de implementar processos de organização da informação para melhorar a estrutura informacional do LAB1 e facilitar o acesso as informações equipe do laboratório.

Palavras-Chave: Diagnóstico, situação do acervo, gestão de informação

\begin{abstract}
It is an article whose objective is to present in detail the situation of LAB1 concerning the organization of information and infrastructure resources available to develop research activities in a biochemistry laboratory. This study had a vision directed to the information management of this laboratory. To understand the situation of the laboratory concerning the information resources, we have to identify and evaluate the documents produced and received, the existing procedures for data management, the difficulties and facilities of the internal team to deal with the knowledge produced, and mainly in verifying how information is recording and recovering from generating new knowledge For this study, the authors used methodological procedures: bibliographic survey, the definition of the data collection instrument, the collection, and the organization of the collected data. The authors have concluded that the diagnosis made shows the need to implement information organization processes to improve the information structure of LAB1 and facilitate access to information from the laboratory staff.
\end{abstract}

Keywords: Diagnosis, status of the collection, information management 


\section{INTRODUÇÃO}

A compreensão da situação que se encontra determinada empresa ou instituição é uma necessidade para propor ações com vistas a implementar mudanças. As mudanças são alterações ou adequações requeridas nas organizações de todos os tipos e podem ocorrer em produtos, serviços ou processos que resultam em transformações no ambiente interno ou externo. Os processos representam uma sequência de atividades necessárias para a realização de uma determinada tarefa. A análise desta sequência em determinado ambiente organizacional possibilita conhecer as melhorias necessárias para alterar ou aperfeiçoar os pontos que merecem atenção.

Mesmo ciente da necessidade de mudanças pode-se afirmar que muitas vezes os atores organizacionais não têm certeza sobre o caminho que deve ser seguido para buscar as alterações necessários aos processos. O diagnóstico é uma ferramenta que permite às instituições de qualquer natureza conhecer detalhadamente sua situação e fornece os elementos para se pensar em implementar, posteriormente uma avaliação das possíveis soluções. Para pensar em estruturar as informações necessárias acerca dos processos referentes a um sistema de informação é relevante considerar os elementos da situação instalada.

\subsection{A gestão da informação e o diagnóstico em unidades de informação}

O diagnóstico organizacional permite a coleta de dados acerca da organização alvo de análise com fins de buscar soluções para os problemas identificados. Em relação ao assunto Grocco e Guttman (2005) afirmam que o diagnóstico organizacional busca identificar e investigar os problemas existentes nas organizações, auxiliando da melhor forma quais são os procedimentos que devem ser aplicados e testados numa tentativa de solução destes problemas. Em relação ao objetivo de um diagnóstico organizacional Chiavenato (2006, p. 438), afirma que o diagnóstico "inclui técnicas e métodos para descrever o sistema organizacional, as relações entre seus elementos ou subsistemas e as maneiras para identificar problemas e assuntos importantes".

As organizações de toda natureza demandam por informação para a realização de suas atividades diárias e para reduzir o nível de incertezas. Neste sentido, observouse que a necessidade de implementar ações para a gestão da informação é premente como apontado por Valentim et al. (2005). Os autores reforçam a importância da gestão de informação e relacionam o sucesso das organizações ao uso de informações confiáveis 
e consistentes para a tomada de decisão. Considerando este aspecto pode-se resgatar Borko (1991) citado por Capurro \& Hjorland (2007) que associa dentre outras características a informação como "coisa". Neste sentido a informação se apresenta como dados, documento ou conhecimento registrado. Também poderia ser intangível e, neste caso, considera-se a Informação como conhecimento

Dias (2002) aponta que autores como Choo (1998) e Davenport (1994) mostram a importância de aspectos relevantes do uso da informação baseado no conhecimento. Eles citam exemplos de produtos, serviços e até a elaboração de sistemas de informação, que podem ser desenvolvidos para subsidiar os vários departamentos de uma empresa, através do uso de informação coletada e estruturada com base nos conhecimentos de especialistas, sua formação e experiências tanto no ambiente interno quanto externo.

Os documentos encontrados nas instituições têm forte relação com as atividades desenvolvidas por estas organizações. Como tal referem-se às evidências destas atividades, são usados como provas das transações realizadas e podem ser portadores de informação para pesquisas. As empresas utilizam as informações e os documentos para a realização de transações financeiras e comerciais, para produzir bens e serviços, para fazer pesquisa, gerar ou aperfeiçoar novos produtos e quaisquer outras tarefas relacionadas às atividades meio e atividades finalísticas. Davenport (1998) ressalta que a gestão da informação trata a informação para além de associá-la aos recursos de tecnologia e que a informação demanda por estão em todas as esferas organizacionais Destaca-se que para o desenvolvimento de sistemas de informação apresentar um retrato da situação e do contexto onde esse sistema está inserido é útil e necessário para subsidiar o planejamento e a estruturação das informações e das fontes de informação. Esta preocupação está relacionada com a necessidade de ter acesso a todos os recursos de informação produzidos pelas instituições por diversas razões: conhecer o acervo documental produzido, usar os documentos e as informações contidas para subsidiar a tomada de decisão e para prestar contas, entre outros usos. O diagnóstico possibilita o conhecimento do retrato de uma determinada situação levantada a partir da análise de vários elementos, que podem ser verificados utilizando entrevistas ou registrando em formulários próprios.

A realização do diagnóstico permite conhecer os elementos relacionados à gestão da informação e a identificação de problemas. Nesta linha apresentam-se a seguir algumas definições para o termo diagnóstico: 
Cornelsen \& Nilli (2006, p. 116) afirmam que o diagnóstico levanta os elementos necessários que estão relacionados ao gerenciamento da informação e destaca soluções que priorizam mudança no tratamento dos documentos gerados e que são arquivados de maneira equivocada.

Ferreira \& Melo (2008) por sua vez destacam que o diagnóstico possibilita levantar os problemas das atividades arquivísticas. Para as autoras o diagnóstico é um instrumento auxiliar que torna possível identificar todos os problemas relacionados ao desenvolvimento das atividades arquivísticas, mediante pesquisas realizadas nas instituições responsáveis pela produção e acumulo dos documentos e, principalmente no próprio arquivo.

A elaboração do diagnóstico analisado neste estudo refere-se à primeira etapa do Plano de Trabalho desenvolvido para organização das informações, análise da estrutura e dos dados de um laboratório de pesquisa em Biologia. O referido laboratório não será citado nominalmente por questões de confidencialidade e será referido apenas como LAB1. Além disto, as informações coletadas para a realização deste diagnóstico foram usadas para subsidiar a elaboração de um projeto de pesquisa para propor uma metodologia de organização e análise da estrutura e dos dados de um Laboratório de Pesquisa em Bioquímica.

Este diagnóstico tem como objetivo apresentar detalhadamente a situação do LAB1 em relação à organização dos recursos de informações existentes no laboratório e de infraestrutura que estão disponíveis para o desenvolvimento das atividades de pesquisa com venenos e toxinas animais realizadas no mesmo.

Este estudo foi realizado especificamente com um propósito direcionado para a gestão de informações do laboratório citado e tem como objetivo principal buscar e avaliar as informações sobre os documentos produzidos e recebidos, a situação em relação à gestão dos dados, a gestão dos recursos informacionais, dificuldades e facilidades da equipe interna para lidar com o conhecimento produzido, com os insumos e amostras utilizadas nos experimentos realizados diariamente pelos pesquisadores e, principalmente na verificação de como se dá o registro das informações e à sua recuperação para gerar novos conhecimentos 


\section{METODOLODOGIA}

Para este estudo, foram utilizados os seguintes procedimentos metodológicos: levantamento bibliográfico, a definição do instrumento de coleta de dados; a coleta de dados e a organização dos dados coletados.

O instrumento para coleta de dados utilizado para o registro das informações do laboratório, Apêndice 1, será detalhado na próxima seção. Os dados que subsidiaram a elaboração deste diagnóstico foram extraídos dos levantamentos realizados no período de novembro a dezembro de 2013 com o objetivo de conhecer a situação do Laboratório em relação aos recursos de informação disponíveis e às ações de organização desses recursos. Estes levantamentos foram efetuados em dois momentos: Internamente, nas instalações do LAB1, durante a visita técnica a todas as áreas. E, uma coleta de dados realizada posteriormente com o envio, por e-mail, do formulário de coleta de dados encaminhado a dez alunos de graduação e de pós-graduação que trabalhavam à época no Laboratório.

A visita técnica foi realizada em dezembro de 2013 acompanhada pela coordenadora do Laboratório e teve como objetivo conhecer as instalações físicas e a infraestrutura do Laboratório, seu campo de atuação e seus recursos de informação. A coleta de dados com a equipe de alunos do LAB1 foi realizada na ocasião com o envio do questionário por e-mail.

\subsection{A primeira etapa: a coleta de dados}

Para a coleta de dados no LAB1 foi construído um formulário chamado de "Diagnóstico" (Apêndice 1), instrumento utilizado para o registro das informações levantadas na visita técnica. Esse instrumento foi organizado em nove grandes tópicos: (i) Laboratório; (ii) Condições físicas do laboratório; (iii) Mobiliário e Equipamentos; (iv) Acervo; (v) Informações sobre projetos e agências de fomento; (vi) Fontes de informação; (vii) Necessidades de informação de cada pesquisador; (viii) Parcerias e (ix) Insumos, amostras e materiais de consumo que estão detalhados a seguir:

1) Laboratório: identificar os dados referentes a contato, unidade e endereço do laboratório.

2) Condições físicas do laboratório: identificar informações como layout da sala, tamanho e aspectos físicos como iluminação, dados sobre instalações elétricas. 
3) Mobiliário e Equipamentos: identificar informações sobre tipo de equipamentos, quantidade de equipamentos e móveis, como é feita sua identificação, métodos e frequência de uso.

4) Acervo: identificar informações sobre forma de organização, existência de informações confidenciais no laboratório, tipos de informações que são armazenadas nos cadernos de laboratório, quantidade, forma de identificação, a estrutura do caderno, local de armazenamento e localização da informação desejada, quais as dificuldades encontradas para acessar as informações, produção cientifica do laboratório, sua tipologia (tese, dissertação, relatórios, artigos, etc.) e quantidade.

5) Informações sobre projetos e agências de fomento: registrar informações sobre projetos de pesquisa executados no laboratório e as agências de fomento que financiam os mesmos, onde o laboratório busca informação, quem são os clientes de informação do laboratório, se existem documentos sobre o assunto, quem são os envolvidos nos projetos e como são controladas as bolsas de estudo.

6) Fonte de informação: registrar informações sobre o controle das fontes de informação usadas no laboratório sua tipologia (tese, dissertação, relatórios, artigos) e referências.

7) Necessidades de informação de cada pesquisador: registrar detalhes sobre as necessidades informacionais dos pesquisadores do laboratório.

8) Parcerias: identificar quem são os parceiros do laboratório e como é feito o compartilhamento de informação/documentos com esses parceiros.

9) Insumos/ amostras e materiais de consumo: coletar informações sobre a gestão do estoque, tipos de insumos ou amostras, marcas, fornecedores, quantidade e data de validade dos insumos.

A base de análise usada para a construção este diagnóstico foi os dados coletados durante a visita técnica, bem como as informações dos formulários preenchidos pela equipe de alunos de graduação e de pós-graduação que desenvolvem atividades de pesquisas no LAB1.

\section{APRESENTAÇÃO DOS DADOS}

Para facilitar o entendimento da situação do LAB1 em relação à gestão de recursos informacionais e posteriormente a definição das ações de melhorias que foram 
propostas, optou-se por mostrar os dados organizados agrupados da seguinte forma: Informações sobre a documentação; Informação sobre o local de armazenamento; Informação sobre Recursos Humanos e materiais (mobiliário e material).

\subsection{Informações sobre a documentação}

A coleta de dados possibilitou a identificação dos diversos recursos existentes no laboratório. Tais recursos foram identificados como mobiliário constituído por geladeiras e freezers, armários, computadores e equipamentos específicos de um laboratório biológico, tal como listado no anexo 1. Observou-se que os freezers e geladeiras armazenam os reagentes, líquidos e materiais de usuários. Foram identificados sete armários no total que armazenavam todo o material de uso da equipe do laboratório.

O item referente a acervo identificado no formulário de coleta de dados foi pensado com a ideia de mapear o acervo documental/ informacional do laboratório. Para identificar esse acervo foi verificado como os recursos de informação estavam organizados e disponibilizados no laboratório. Além disso verificou-se também, a existência de informações confidenciais. Neste caso, a ideia foi verificar quais eram estas informações, como e onde ficavam armazenadas e quem tinha acesso a tais informações.

Observou-se que a documentação do laboratório pode ser caracterizada por um acervo diversificado como a produção científica do laboratório composta por artigos publicados em periódicos, teses e dissertações, trabalhos apresentados em eventos técnico-científicos, documentos referentes à participação em bancas de concurso, qualificação de mestrado e de doutorado, informações sobre os experimentos realizados internamente, sobre as parcerias, sobre os projetos, sobre as agências de fomento e sobre os bolsistas. Neste sentido, observou-se que há uma quantidade de informações referentes às atividades específicas do laboratório que necessitam de uma estruturação e de uma organização de tal forma que seja possível agilizar o acesso da equipe e otimizar seu uso.

Enfim, verificou-se que há uma quantidade muito grande de informações que trafegam pelo laboratório e em algumas situações estas informações são de acesso mais fácil para aqueles que lidam diretamente com ela. Merece destaque, também, a dificuldade em se compartilhar informações em rede para que todos os membros do 
laboratório possam usufruir os recursos informacionais existentes. Neste sentido, há uma preocupação em criar uma metodologia para organizar as informações oriundas das diversas fontes e torná-las acessíveis para a geração de novos conhecimentos. Com tudo isto, observou-se que há no LAB1 oportunidades de melhorias em relação à organização da informação de forma geral e mais especificamente com a proposição de metadados para a representação e a gestão das informações sobre protocolo usados nos experimentos do laboratório, bem como a possibilidade de implementação de ferramentas para otimizar o seu armazenamento e a sua recuperação sempre que houver necessidade.

A coleta de dados possibilitou a identificação e a função dos diversos recursos existentes no laboratório. Tais recursos foram identificados como mobiliário constituído por geladeiras e freezers, armários, computadores e equipamentos específicos de laboratório, tal como listado no Apêndice 1. Observou-se que os freezers e as geladeiras são usados para o armazenamento de reagentes, de líquidos diversos usados nos experimentos de pesquisas realizados, assim como de materiais de usuários do LAB1. Além destes equipamentos foram identificados também armários (sete no total) usados para o depósito de todo o material de uso da equipe do laboratório.

O item referente a acervo identificado no formulário de coleta de dados foi pensado para o registro de dados do acervo documental e informacional do laboratório. Logo, neste quesito foi verificado como as informações são organizadas e são disponibilizadas, sobre a existência de informações confidenciais e neste caso quais são elas, como e onde ficam armazenadas e quem pode ter acesso.

Observou-se que a documentação é formada por um acervo diversificado como a produção científica do laboratório composta por artigos publicados em periódicos, teses e dissertações, trabalhos apresentados em eventos técnico-científicos, documentos referentes à participação em bancas de concurso, qualificação de mestrado e de doutorado, informações sobre os experimentos realizados internamente, sobre as parcerias, sobre os projetos, sobre as agências de fomento e sobre os bolsistas. Observouse que há uma quantidade expressiva de informações referentes às atividades específicas do laboratório que necessitam de estruturação e de organização para um posterior acesso da equipe interna ao LAB1.

Enfim, observou-se que há uma quantidade muito grande de informações que trafega pelo laboratório, mas em algumas situações estas informações são de acesso mais 
fácil para aqueles que estão envolvidos diretamente na realização de pesquisas com as quais estão envolvidas. Merece destaque, também, a dificuldade de compartilhar informações em rede para que toda a equipe do LAB1 possa usufruir dos recursos informacionais existentes. Para atender a este ponto, uma preocupação é criar uma metodologia que possa organizar as informações oriundas das diversas fontes e tornálas acessíveis para a geração de novos conhecimentos no laboratório. Com tudo isto, observou-se que há no LAB1 oportunidades de melhorias em relação à organização da informação, a possibilidade de implementação de ferramentas para otimizar o seu armazenamento e a sua recuperação sempre que houver necessidade.

\section{Acervo informacional armazenado no LAB1}

Em relação ao acervo documental existente no LAB1 observou-se que há uma diversidade de tipos documentais e uma grande quantidade de equipamentos que contém informações sobre conteúdo específico referente a insumos e amostras usados para a realização das diversas atividades desenvolvidas pelas equipes de pesquisadores; produção científica do laboratório. Este acervo contém as seguintes informações:

- Amostras e organismos utilizados nos experimentos

- Métodos e protocolos experimentais

- Insumos e materiais de laboratório

- Cadernos de laboratório

- Material bibliográfico formado por: artigos, trabalhos apresentados em eventos técnicos da área, referências, teses e dissertações

- Projetos de pesquisa

- Informação sobre a equipe

- Informação sobre agências de fomento

- Informação sobre equipamentos

- Informação sobre parcerias e contatos científicos

Segue uma descrição mais detalhada das informações relacionadas a este acervo:

Experimentos, materiais e organismos: A informação sobre os experimentos realizados, assim como os insumos e organismos utilizados estão quase que invariavelmente registrados nos chamados cadernos de laboratório. Os cadernos são individuais e cada 
estudante ou pesquisador registra livremente e de forma personalizada as informações referentes aos experimentos relacionados à pesquisa em curso. Durante a visita técnica percebeu-se que um caderno de laboratório contém registros de muito valor para o pesquisador, pois contém dados das análises durante o período das pesquisas, assim como podem necessários para usos futuros no caso da realização de novos produtos, para repetição dos experimentos por outros pesquisadores, facilitando a continuidade da pesquisa e, por fim os dados registros podem ser muito úteis para um registro de patente. Por esse motivo, observou-se, ainda que alguns resultados são registrados em arquivos de computador do tipo planilhas eletrônicas e contém imagens. Notou-se que o uso de um padrão para estes registros ou para a forma de nomear os arquivos pode facilitar o acesso às informações. Outro aspecto observado é que estabelecimento dos POPS (Procedimento Operacional Padrão) e das BPLs (Boas Práticas de Laboratório) podem auxiliar na sistematização e na padronização e organização de processos referentes às informações dos laboratórios.

Materiais e organismos: Como os laboratórios compram material para consumo e organismos que duram bastante tempo e são utilizados muitas vezes, é preciso manter um controle do que há em estoque, qual marca é utilizada com sucesso, quando são feitas as compras, datas de validade e coisas deste tipo. Esta informação é mantida em cadernos de almoxarifado ou fichas de papel. Em relação a este controle, observou-se que há oportunidades de melhorias para facilitar a gestão destes insumos, visto que é rara a presença de sistemas de controle de estoque, ainda que sejam manuais.

Métodos e Protocolos: Os métodos utilizados nos experimentos estão em geral registrados nos cadernos de laboratório. Nos locais mais organizados há uma pasta de protocolos que serve como guia de referência para todos, onde os protocolos estão organizados em pasta do tipo catálogo. Como a maioria dos protocolos é retirada de artigos científicos, é listada a referência do artigo e somente as modificações do protocolo são anotadas.

Informação sobre a equipe: Apenas o pesquisador principal ou chefe do laboratório controla quais são os estudantes e técnicos têm acesso às instalações. Os alunos são matriculados pelas universidades e os técnicos são funcionários alocados em cada laboratório. Não há um sistema formal de controle da equipe, de suas atividades e sua movimentação nas entradas e saídas, bem como dos produtos das pesquisas realizadas, assim como teses e dissertações produzidas. 
Material bibliográfico: formado por: teses, artigos, trabalhos apresentados em eventos técnicos da área, listas de referências. Observou-se que também, neste caso, o controle é informal da produção. As referências bibliográficas são de uso individual e cada aluno ou pesquisador tem seu conjunto pessoal e faz uma gestão livre deste material. Membros da mesma equipe podem optar por compartilhar com os colegas este tipo de material.

Agências de fomento: $O$ controle dos projetos é feito inteiramente pelo Chefe do laboratório. Em alguns casos há um aluno do laboratório que controla parte do processo, mas não há um procedimento formal que auxilia o arquivamento desses documentos. $\mathrm{O}$ controle dos recursos é feito pelas próprias agências ou fundações intervenientes.

Observou-se que o LAB1 possui uma quantidade muito grande de informações (Figura 1) que estão registradas de forma dispersa em vários locais. Em alguns casos as informações estão sob a responsabilidade dos estudantes ou registradas de forma personalizadas nos cadernos de laboratório. Neste último caso, percebeu-se que o caderno de laboratório é uma fonte de informação de extrema importância, pois ele contém todos os registros acerca de um experimento, protocolo usado, referências, datas, nome do pesquisador, observações, resultados, entre outras informações. Segundo informações, os cadernos de laboratório ficam sob a responsabilidade do estudante responsável que tem liberdade para organizar a forma de fazer o registro das informações. Além disto, ressalta-se que estes cadernos têm um valor de prova e, logo são considerados como documentos do laboratório e podem ser usados em situações que exijam a comprovação da autenticidade de informações, inclusive em disputas judiciais. Entretanto, observou-se que estes cadernos são organizados de forma personalizada e que, o acesso é restrito ao próprio estudante.

Em relação à forma como as informações são disponibilizadas verificou-se que o laboratório não possui regras muito claras sobre a organização das informações.

Questionados sobre a existência de informações confidenciais, sua forma de armazenamento e acesso verificou-se que o LAB1 possui informações desta natureza e que toda a equipe interna pode acessar estas informações desde que o laboratório seja avisado. Com isto é necessário verificar ainda, quais são estas informações e com quem elas podem ser compartilhadas. 


\subsection{Informação sobre o local de armazenamento}

O espaço físico do LAB1 é composto por mesas, bancadas, equipamentos específicos de laboratório de biologia, mesas, cadeiras, armários, geladeiras e freezers, computadores, entre outros mobiliários e equipamentos.

\subsection{Informação sobre as fontes de informação}

A ideia de pensar nas fontes de informação no LAB1, durante o levantamento de informações foi compreender a sua possibilidade de uso pela equipe interna. Para isto foram levantadas informações considerando os seguintes aspectos relacionados às fontes de informação: sua tipologia, como é feito o controle das fontes usadas no laboratório, uma possível relação de referências do material usado com o objetivo de armazenamento futuro e por fim analisar se existem ou não ações de compartilhamento de informações entre a equipe. Quando a equipe interna foi questionada sobre como é feito o controle das fontes usadas no laboratório, um dos participantes respondeu que às fontes impressas estão disponíveis para consulta interna e as digitais foram copiadas para os computadores pessoais. Quatro alunos disseram que não há nenhum controle sobre as fontes de informação e um não respondeu à questão sobre as necessidades de informação. A Figura 1 ilustra os diversos recursos de informação identificados no Laboratório analisado.

Em relação às referências, por exemplo, foi informado por um dos bolsistas que “todas as referências encontram-se disponíveis on-line e é e responsabilidade do usuário o seu armazenamento da forma que melhor o convir" (bolsista) o que denota que não há uma orientação para a equipe de bolsistas em relação ao uso das fontes de informação. 
Figura 1 - Mapa dos recursos do Laboratório

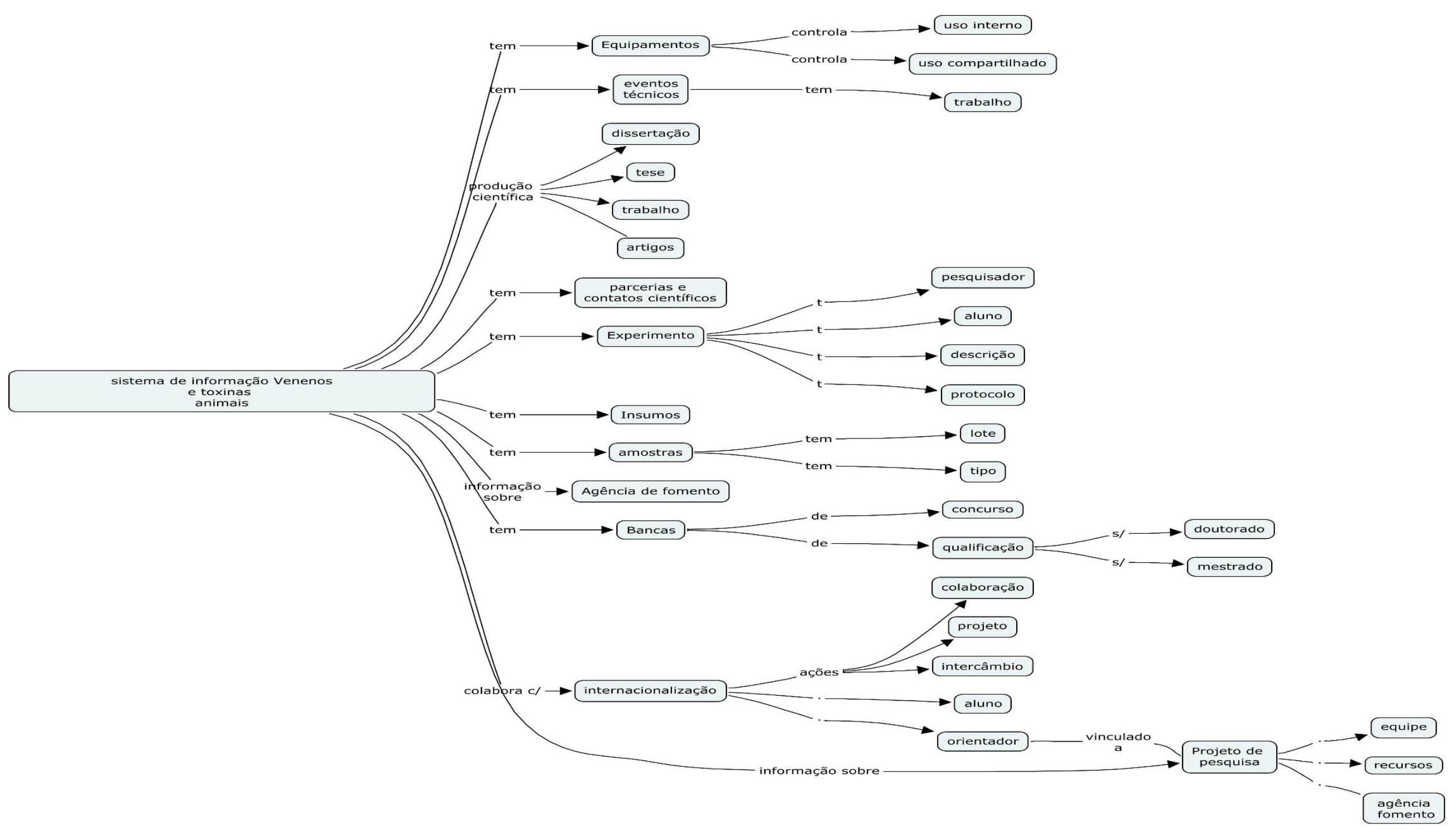




\subsection{Necessidades de informação}

O levantamento sobre as necessidades de informação da equipe interna do laboratório teve como objetivo verificar a compreensão geral sobre quais informações são requeridas para a realização do trabalho diário do LAB1. Este item não foi respondido por todos os membros do laboratório. As respostas obtidas foram: conhecimento dos reagentes e equipamentos disponíveis e sua política de uso, conhecimento sobre as questões éticas que envolvem o uso de animais. Outro aluno mencionou que o laboratório é carente de informações e com isto pode-se deduzir que a necessidade de informação dos pesquisadores não tem sido totalmente satisfeita. Segundo este aluno falta informação sobre quase tudo, como por exemplo, sobre as teses e dissertações disponíveis no laboratório, informações que não estão funcionando, uma catalogação dos reagentes, entre outros. Além disto, foi listada a ausência de informações sobre os reagentes disponíveis e onde eles estão alocados, sobre as políticas do laboratório sobre descarte de materiais e utilização de equipamentos, relação dos equipamentos disponíveis. Dois alunos não responderam.

\subsection{Recursos Humanos}

Em relação aos recursos humanos observou-se que há um servidor técnicoadministrativo alocado no laboratório, além dos estudantes e pesquisadores. Entretanto, esta equipe está envolvida diretamente com a realização das atividades fins do LAB1, não dispondo de tempo e de formação para a organização das informações. Apesar deste aspecto, a organização dos materiais e das informações fica sob a responsabilidade de toda a equipe.

\section{CONSIDERAÇÕES FINAIS}

Este artigo descreveu detalhadamente a situação informacional de um laboratório de pesquisa em Biologia. Observou-se que o intenso trabalho realizado nos laboratórios de biologia, a demanda exaustiva por resultados, a publicação de artigos, participação em eventos científicos, desenvolvimento de produtos, pela geração de novos conhecimentos não permite à equipe dispor de tempo para a organização das informações. Entretanto, a informação e o conhecimento são insumos fundamentais para a realização das pesquisas em qualquer laboratório. E por esse motivo, percebeu-se a importância de atender às necessidades de 
informação da equipe do LAB1 nos requisitos de organização dos recursos de informação, bem como em ferramentas que auxiliem a equipe do LAB1 na gestão da informação.

\section{AGRADECIMENTOS}

Os autores agradecem ao laboratório da UFMG utilizado no diagnóstico e as agências de fomento CNPq e FAPEMIG pelo auxílio financeiro.

\section{REFERÊNCIAS}

CAPURRO, R. \& HJORLAND, B. O conceito de informação. Perspectivas em Ciência da Informação, v.12, n.1, p.148-207, jan/abr.2007.

CHIAVENATO, I. Recursos humanos: o capital humano das organizações. 8. ed. São Paulo: Atlas, 2006. $\mathrm{CHOO}, \mathrm{C} . \mathrm{W}$. (Ed.). Information management for the intelligent organization: the art of scanning the environment. Hedford: New Jersey; Learned Information, 1998.

CORNELSEN, Julce Mary; NELLI, Victor José. Gestão integrada da informação arquivística: o diagnóstico de arquivos. Arquivística.net), Rio de Janeiro, v.2, n. 2, p 70-84, ago./dez. 2006. Disponível em : www.arquivistica.net . Acessado em 01/10/2020

CROCCO e GUTTMANN, Luciano e Erik. Consultoria Empresarial. São Paulo: Saraiva, 2005.

DAVENPORT, T. Ecologia da informação: porque só a tecnologia não basta para o sucesso na era da informação. São Paulo: Futura, 1998

DIAS, Célia da Consolação. Monitoração Ambiental: um estudo de caso em Empresas do setor de Telecomunicações. 2002. 127fls. Dissertação (Mestrado em Ciência da Informação). Belo Horizonte: UFMG.

FERREIRA, Lucienne da Costa; MELO, Denise Gomes Pereira de. Diagnóstico de arquivos: instrumento de ação efetiva na gestão documental.

FÓRUM INTERNACIONAL DE ARQUIVOLOGIA, 1 - UEPB - Campus V - João Pessoa, 25 a 27 de novembro de 2008 .

VALENTIM, M. L. P. et al. Pesquisa em inteligência competitiva organizacional: utilizando a análise de conteúdo para a coleta de dados e análise de dados - Parte II. Transinformação, Campinas, v. 17, n. 3, p. 253-270, set./dez. 2005. 


\section{APÊNDICE 1 - FORMULÁRIO DE COLETA DE DADOS}

\section{FORMULARIO DE COLETA DE DADOS - LABORATORIO DE BIOQUÍMICA - LAB1}

\begin{tabular}{|c|c|c|}
\hline \multicolumn{3}{|c|}{ LABORATORIO DE BIOQUÍMICA - LAB1 } \\
\hline Unidade: & Endereço: & \\
\hline \multicolumn{3}{|l|}{ Contato } \\
\hline Nome: & Telefone: & \\
\hline \multicolumn{3}{|c|}{ Condições físicas do laboratório } \\
\hline \multicolumn{3}{|c|}{$\begin{array}{l}\text { Layout da sala: } \\
\text { Aspectos físicos: } \\
\text { Iluminação: }\end{array}$} \\
\hline \multicolumn{2}{|l|}{ Instalações } & elétricas: \\
\hline \multicolumn{2}{|l|}{ Instalações } & elétricas: \\
\hline \multicolumn{3}{|c|}{ Mobiliário e Equipamentos p/armazenamento } \\
\hline \multicolumn{3}{|c|}{$\begin{array}{l}\text { Freezers: quantidade: } \\
\text { Conteúdo: }\end{array}$} \\
\hline \multicolumn{2}{|c|}{ Como é feito a identificação? } & quantidade: \\
\hline \multicolumn{3}{|c|}{ Conteúdo: } \\
\hline \multicolumn{2}{|c|}{$\begin{array}{l}\text { _Como é feito a identificação? } \\
\text { Armários: }\end{array}$} & quantidade: \\
\hline \multicolumn{3}{|l|}{ Conteúdo: } \\
\hline $\begin{array}{l}\text { Como é feito a ide } \\
\text { Computadores } \\
\text { Conteúdo: }\end{array}$ & & quantidade: \\
\hline \multicolumn{2}{|l|}{ Outros } & equipamentos: \\
\hline \multicolumn{3}{|l|}{$\begin{array}{l}\text { Quantidade: } \\
\text { Conteúdo: }\end{array}$} \\
\hline \multicolumn{3}{|l|}{ ACERVO } \\
\hline \multicolumn{3}{|c|}{ Como a informações são organizadas e disponibilizadas? } \\
\hline \multicolumn{3}{|c|}{ Existem informações confidenciais no laboratório? } \\
\hline \multicolumn{3}{|l|}{ Quais? } \\
\hline \multicolumn{3}{|c|}{ Onde elas ficam armazenadas? } \\
\hline Quem pode ter ac & nações? & \\
\hline
\end{tabular}




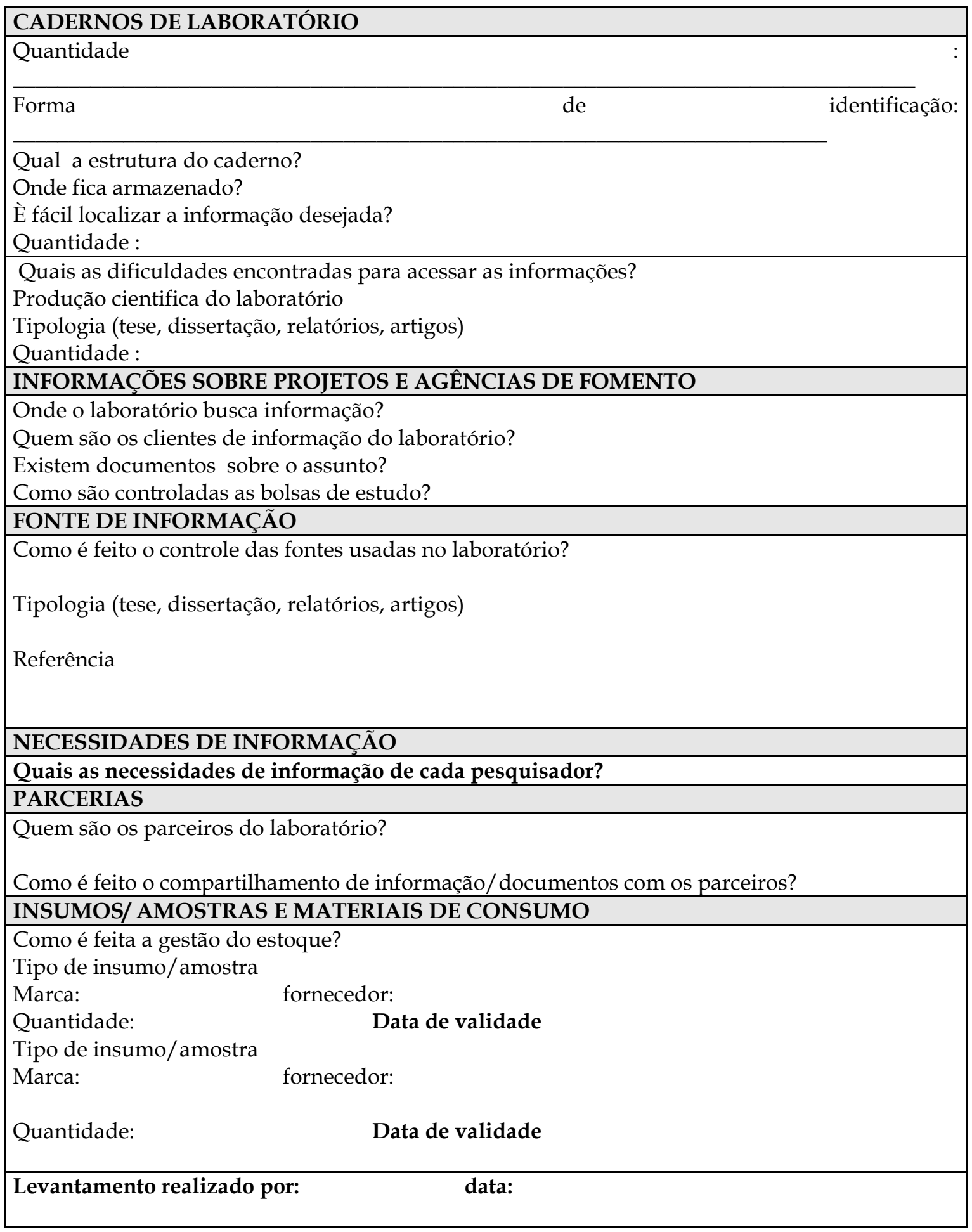

